\title{
Metabolic characteristics of individuals at a high risk of type 2 diabetes - a comparative cross-sectional study
}

\author{
Josefin Henninger ${ }^{*}$, Araz Rawshani, Ann Hammarstedt and Björn Eliasson
}

\begin{abstract}
Background: Type 2 diabetes (T2D) is associated with substantial morbidity and mortality. Individuals with a family history of T2D are at an increased risk of developing the disease. The aim of this study was to assess metabolic differences between first-degree relatives (FDR) of T2D patients and persons with no known family history of T2D (non-FDR).

Methods: In 200 FDR and 73 non-FDR, we compared anthropometrics, glucose tolerance status, different measurements of insulin secretion, insulin resistance, as well as blood lipids and other blood analyses.

Results: In the FDR group, 30 individuals had impaired glucose tolerance or T2D. Among the non-FDR, two individuals had impaired glucose tolerance. In unadjusted data, the FDR were older, had stronger heredity for coronary heart disease, lower body mass index and weight, higher OGTT plasma glucose concentrations, and impaired insulin secretion (all $p<0.05$ ). Using propensity score, we matched the groups, resulting in significantly stronger heredity of coronary heart disease, higher OGT plasma glucose at 60 and 90 min, larger glucose area under curve during the OGT and higher serum creatinine among the FDR. Using least squares means, OGTT glucose at 60 and $120 \mathrm{~min}$, as well as the area under curve, and OGT insulin levels at $60 \mathrm{~min}$ were significantly higher. Body mass index was negatively correlated with insulin sensitivity (MI) and positively correlated with HOMA- $\beta$, a measurement of insulin secretion.

Conclusions: We show that FDR are more likely to have impaired glucose tolerance and display higher OGT plasma glucose and insulin, indicating an unfavorable metabolic profile. We conclude that OGTT is a simple and yet informative metabolic assessment in the FDR group. In both groups, we saw a negative correlation between body mass index and Ml, confirming the role of body mass index in insulin resistance.
\end{abstract}

Keywords: Type 2 diabetes, First-degree relatives, Cross-sectional study

\section{Background}

Type 2 diabetes mellitus (T2D) is a heterogeneous disease characterized by chronically elevated plasma glucose levels, as well as disturbances in the metabolism of lipids, carbohydrates and protein $[1,2]$. It is intimately associated with obesity and the metabolic syndrome [2-4], which are major risk factors of cardiovascular disease [5], the number one cause of death globally today $[4,6]$.

\footnotetext{
*Correspondence: josefin.henninger@gu.se

The Lundberg Laboratory for Diabetes Research, Department of Molecular and Clinical Medicine, the Sahlgrenska Academy at the University of Gothenburg, 41345 Gothenburg, Sweden
}

The pathophysiology behind T2D has been extensively studied and there is consensus in the research community today that the pathophysiology indeed is multifactorial $[1,2,7]$. DeFronzo et al. summarizes how consensus has grown from acknowledging functional disturbances in pancreatic islet $\beta$-cell (ie insulin secretion) and the liver and muscle cells (ie insulin action), to today include well documented perturbations in adipose tissue metabolism and morphology, gut hormone regulation (ie incretin action), kidney function and pancreatic $\alpha$-cell function, as well as central nervous system neurotransmitter dysfunction [1]. 
Lifestyle factors, in particular a hypercaloric diet, cigarette smoking and a sedentary lifestyle, in combination with genetic factors, are considered central underlying causes $[8,9]$. In spite of only weak associations between specific genes and the development of T2D, first-degree relatives (FDR) of T2D patients are clearly at an elevated risk of developing the same disease, proportionate to the number of family members affected $[2,3$, $10,11]$.

Previous studies have shown that FDR display unfavorable anthropometrics and metabolic characteristics in spite of normoglycemia, however less refined methods have been used [12-17]. The aim of this study is to assess anthropometric and metabolic differences, using state of the art methods, between a group of healthy FDR and a group of healthy persons without known family history of diabetes, recruited from the background population.enzyme-linked immunoassay (ELISA) technique. Serum insulin was analyzed at the Wallenberg laboratory using a radioimmunoassay technique (Pharmacia, Uppsala, Sweden). Plasma insulin was measured at the University of Tübingen, Germany, by micro-particle enzyme immunoassay (Abbott Laboratories, Tokyo, Japan). A conversion factor was used to compare the two. Serum adiponectin was measured by a human adiponectin ELISA-kit (B-Bridge International, Sunnyville, CA, USA). Serum LDL cholesterol was calculated using the Friedewald equation [18]. At this visit the subjects also filled out a questionnaire regarding lifestyle and family history of diabetes. High heredity for T2D was defined as having more than one FDR with the disease. Level of stress was subjectively assessed.

At a second visit, an OGTT was performed. We used the WHO criteria for IGT and T2D [19]. The OGTT was performed by oral administration of $75 \mathrm{~g}$ of liquidized glucose at a fasting state and subsequent intravenous blood glucose samples (in $\mathrm{mmol} / \mathrm{L}$ ) at $30,60,90$ and $120 \mathrm{~min}$. The $120 \mathrm{~min}$-value was used to assess glucose tolerance status as NGT, IGT or T2D [20]. To further assess glucose tolerance status, the concentrations of glucose and insulin concentrations were plotted over time. An area under curve (AUC) of glucose and insulin was calculated using the trapezoid method [21].

We also analyzed serum (in the non-FDR group) and plasma (in the FDR group) insulin levels ( $\mathrm{pmol} / \mathrm{L}$ ) at 0,30 , 60,90 and 120 min. HOMA- $\beta$ was calculated using fasting glucose and fasting insulin levels [22]. The formula looks as follows:

HOMA-beta $\%=\frac{\text { fasting plasma insulin }\left(\frac{p m o l}{L}\right) \times 20 \div 6.945}{\text { fasting plasma glucose }\left(\frac{\text { mmol }}{L}\right)-3.5}$

IGI ( $\mathrm{pmol} / \mathrm{mmol}$ ) was calculated using the following formula: $\frac{\Delta \text { plasma insulin } \quad\left(\frac{\text { pmol }}{L}\right)}{\Delta \text { plasma glucose } \quad\left(\frac{\text { mmol }}{L}\right)}$
The delta values are the difference between the plasma values at $120 \mathrm{~min}$ and fasting values during the OGTT $[23,24]$.

At the third visit, a hyperinsulinemic, euglycemic clamp - the gold standard and reference method to assess insulin sensitivity - was performed on each subject. A disposition index, derived from IGI from the OGTT and the $\mathrm{M} / \mathrm{I}$-value from the clamp performed at this visit, was also calculated [25], thus providing a $\beta$-cell function estimate superior to the IGI and the HOMAbeta, as it takes insulin resistance into account [26]: $\frac{I G I}{\frac{I}{M / I}}=I G I \times\left(\frac{M}{I}\right)=\frac{p m o l}{m m o l} \times \frac{m g \div k g \div \min }{p m o l \div L}=\frac{m L}{m o l \div \min }$

The clamp was performed in a fasting state by a continuous intravenous infusion of insulin, to suppress endogenous insulin production, and subsequent and variable glucose infusion. Glucose was measured at five min intervals until a steady state was reached, where plasma glucose was clamped at $5.0 \mathrm{mmol} / \mathrm{L}$. At the clamped plasma glucose level, the glucose infusion rate (GIR, ie M-value), divided by lean body mass was calculated as a measurement of the whole body glucose uptake [27]. Also, to account for endogenous insulin levels, an M/I (I being insulin levels during the steady-state phase of the clamp) was also calculated [28].

\section{Statistical analysis}

Analyses were performed using SPSS statistics version 22.0 (Armonk, NY, USA: IBM Corp.), as well as R (The $\mathrm{R}$ Project for Statistical Computing version 3.1.2). Variables were considered normally distributed after visual assessment in a histogram. The results for continuous variables are given as means and SD, and for categorical variables as frequencies. Student's t-test was used to assess statistical significance between groups for continuous variables and for categorical variables Chi-square test was used. A value of $p<0.05$ was considered statistically significant. However, the $p$ values should be interpreted in the light of the exploratory nature of the study and the number of tests performed.

We used linear regression to compare IGI, HOMA- $\beta$, DI, M, MI and plasma insulin and glucose, as well as insulin and glucose AUC, between non-FDR and FDR. Adjustment was made for age, sex, BMI, physical exercise and smoking. To obtain a mean value for each dependent variable, we used least squares means with the same model specifications. Group differences were examined with and without imputation of missing data. Missing data was imputed using the MICE algorithm (we imputed 5 complete data sets).

We also performed a 1-to-1 matching, using a propensity score. We matched each non-FDR subject to one 
FDR subject, conditional on age, sex, and BMI. The resulting matched group consisted of 146 individuals; 73 subjects in each group. We compared the groups directly in order to explore metabolic differences between non-FDR and FDR at the same age, sex and BMI.

Correlations between BMI and fat percent on one hand, and IGI, MI and HOMA- $\beta$ on the other in nonFDR and FDR were also assessed, by use of Spearman's correlation [29-31].

\section{Results}

Two hundred subjects were FDR and 73 were non-FDR. In the FDR group, 170 individuals displayed NGT, 29 had IGT, 6 had IFG (of which 3 also had IGT) and 1 individual fulfilled the T2D criteria. Among the non-FDR, 70 were NGT, 2 individuals had IGT and 1 had IFG.

\section{Crude characteristics}

Clinical, anthropometric and biochemical characteristics of the complete and unmatched two groups are displayed in Table 1 . The FDR were significantly older, displayed stronger heredity for CHD, and had lower BMI and weight, but still similar WHR supporting an increased predisposition for an abdominal adipose tissue profile. They also displayed higher plasma glucose concentrations during the OGTT, including AUC, however at equal insulin concentrations, as well as higher serum HDL and lower LDL. There were non-significant differences in insulin sensitivity ( $p$-values $<0.1$ ), while measurements of insulin secretion showed that the FDR had lower HOMA- $\beta$, higher IGI and DI were not statistically significantly different.

\section{Differences and similarities in the matched set}

Clinical, anthropometric and biochemical characteristics in the matched set, using propensity score, are displayed in Table 2. When comparing the two matched groups of individuals, FDR only had a significantly higher level of heredity for CHD, higher OGTT plasma glucose at 60 and $90 \mathrm{~min}$, as well as AUC, lower serum LDL and higher serum creatinine.

Figure 1 a-d show mean plasma glucose and insulin concentrations during the OGTT in non-FDR and FDR, overall and in the matched set. Elevations in plasma concentration of glucose can be seen throughout the trial, including a larger AUC in the FDR group. AUC for plasma insulin did not differ significantly between groups.

We examined least square means and $95 \%$ confidence intervals for IGI, HOMA- $\beta$, DI, M, MI and OGTT glucose and insulin, including AUC. Means and confidence intervals were derived from the complete cases, whereas $p$ values were pooled over the 5 imputed data sets. OGTT glucose at 60 and $120 \mathrm{~min}$, as well as AUC glucose were significantly higher, and OGTT insulin at 60 min were significantly higher among $\operatorname{FDR}(p<0.05)$. The other examined variables did not reach statistical significance, however insulin and glucose levels tended to be higher in the FDR group throughout the OGTT. Also, IGI and DI both tended to be lower in the FDR group, indicating impaired $\beta$-cell function. These figure have not been included in this publication.

\section{Correlations}

We examined correlations between BMI and fat percent, and IGI, MI, HOMA- $\beta$ in both groups, including all individuals (Fig. 2). In neither of the groups we saw a statistical significant correlation between IGI and BMI or fat percent. BMI was negatively correlated with MI and positively correlated with HOMA- $\beta$ in both non-FDR and FDR, although more strongly in non-FDR. When examining which correlations were present with fat percentage, only a relatively weak negative correlation with $\mathrm{MI}$ in the FDR group and a positive correlation with HOMA- $\beta$ among non-FDR reached statistical significance.

\section{Discussion}

In summary, we found that healthy FDR display higher glucose and insulin levels during an OGTT, higherserum triglycerides and a tendency towards disturbed $\beta$-cell dysfunction, when compared to subjects without a family history of diabetes. Using covariate analysis, we also found that OGTT glucose at 60 and $120 \mathrm{~min}$, aswell as AUC glucose were significantly higher, and OGTT insulin at 60 min were significantly higher, among FDR. We also found that in the FDR group, fat percentage and BMI were both negatively correlated with MI.

A primary finding of this study was the elevated insulin and glucose levels during and OGTT, despite equal insulin resistance levels between groups, and thus confirming the OGTT usefulness in detecting high-risk individuals, despite its simplicity. Our data, both adjusted and unadjusted, showed higher plasma glucose and insulin concentrations, including AUC and iAUC, throughout the OGTT in the FDR compared to non-FDR. Other studies have thoroughly examined OGTT findings in FDR subjects, and have reached similar conclusions. Taheri et al. and Alyass et al. hypothesized, that measurements at $60 \mathrm{~min}$ during an OGTT might detect disturbances not shown at 120 min [10, 12, 13, 32, 33]. In the Baltimore Longitudinal study of ageing, OGTT data, including AUC, from 1400 non-diabetic individuals showed that integrated glucose, ie glucose AUC, as well as glucose concentration at $120 \mathrm{~min}$, were significant predictors of all-cause mortality [34].

In this study, we also aimed to study insulin secretion function and found that there is a tendency, however 
Table 1 Subject characteristics of the complete two groups, unadjusted

\begin{tabular}{|c|c|c|c|}
\hline & Non-FDR & FDR & $p$ \\
\hline N & 73 & 200 & \\
\hline Sex male (\%) & $33(45.2)$ & $81(40.5)$ & 0.576 \\
\hline Age (years) & $36.68(7.99)$ & $40.05(7.16)$ & 0.001 \\
\hline Weight (kg) & $83.93(14.86)$ & $75.98(12.95)$ & $<0.001$ \\
\hline $\mathrm{BMI}\left(\mathrm{kg} / \mathrm{m}^{2}\right)$ & $27.22(3.91)$ & $25.14(3.47)$ & $<0.001$ \\
\hline Fat percent (\%) & $27.87(9.24)$ & $26.42(8.09)$ & 0.218 \\
\hline $\mathrm{DI}(\mathrm{mL} / \mathrm{mol} / \mathrm{min})$ & $3.63(3.56)$ & $2.78(2.88)$ & 0.108 \\
\hline Waist circumference (cm) & $90.37(10.64)$ & $88.39(10.20)$ & 0.169 \\
\hline WHR & $0.85(0.08)$ & $0.86(0.08)$ & 0.334 \\
\hline Diastolic blood pressure (mmHg) & $77.53(8.98)$ & $73.64(9.75)$ & 0.003 \\
\hline Systolic blood pressure (mmHg) & $121.19(14.50)$ & $115.99(12.22)$ & 0.004 \\
\hline High heredity for T2D (\%) & $0(0.0)$ & $7(3.6)$ & $<0.001$ \\
\hline High heredity for CHD (\%) & $9(13.0)$ & $76(29.6)$ & 0.001 \\
\hline Currently smoking (\%) & $7(10.2)$ & $22(11.3)$ & 0.877 \\
\hline Exercise $>4$ times per week (\%) & $41(57.7)$ & $68(35.2)$ & 0.008 \\
\hline High stress level (\%) & $9(13.0)$ & $34(17.7)$ & 0.010 \\
\hline Serum adiponectin (ng/mL) & $8295.29(3551.68)$ & $9064.92(4436.85)$ & 0.233 \\
\hline Serum triglycerides (mmol/L) & $0.91(0.48)$ & $1.03(0.54)$ & 0.131 \\
\hline Serum HDL (mmol/L) & $1.42(0.32)$ & $1.55(0.38)$ & 0.031 \\
\hline Serum cholesterol (mmol/L) & $4.66(0.86)$ & $4.82(0.89)$ & 0.185 \\
\hline Serum LDL (mmol/L) & $3.78(0.80)$ & $2.84(0.84)$ & $<0.001$ \\
\hline Serum creatinine $(\mu \mathrm{mol} / \mathrm{L})$ & $74.91(12.45)$ & $85.86(16.81)$ & $<0.001$ \\
\hline OGTT fasting plasma glucose (mmol/L) & $4.74(0.36)$ & $4.81(0.42)$ & 0.165 \\
\hline OGTT plasma glucose $30 \mathrm{~min}(\mathrm{mmol} / \mathrm{L})$ & $7.55(1.54)$ & $7.99(1.54)$ & 0.044 \\
\hline OGTT plasma glucose $60 \mathrm{~min}(\mathrm{mmol} / \mathrm{L})$ & $6.90(1.90)$ & $7.76(2.12)$ & 0.003 \\
\hline OGTT plasma glucose $90 \mathrm{~min}(\mathrm{mmol} / \mathrm{L})$ & $5.87(1.51)$ & $6.95(1.89)$ & $<0.001$ \\
\hline OGTT plasma glucose $120 \mathrm{~min}(\mathrm{mmol} / \mathrm{L})$ & $5.56(1.37)$ & $6.12(1.59)$ & 0.010 \\
\hline OGTT fasting serum insulin (pmol/L) & $58.77(36.19)$ & $49.20(29.30)$ & 0.048 \\
\hline OGTT serum insulin 30 min (pmol/L) & $489.05(336.05)$ & $412.03(245.01)$ & 0.068 \\
\hline OGTT serum insulin $60 \mathrm{~min}(\mathrm{pmol} / \mathrm{L})$ & $454.58(297.68)$ & $431.35(261.66)$ & 0.572 \\
\hline OGTT serum insulin $90 \mathrm{~min}(\mathrm{pmol} / \mathrm{L})$ & $336.85(257.46)$ & $396.38(297.55)$ & 0.162 \\
\hline OGTT serum insulin $120 \mathrm{~min}(\mathrm{pmol} / \mathrm{L})$ & $305.38(267.73)$ & $318.93(288.16)$ & 0.746 \\
\hline HOMA- $\beta$ (\%) & $150.53(81.27)$ & $118.88(63.90)$ & 0.003 \\
\hline IGI (pmol/mmol) & $194.70(250.13)$ & $136.18(144.01)$ & 0.044 \\
\hline M-value (GIR/kg/min: g/kg/min) & $11.94(4.49)$ & $13.00(4.16)$ & 0.085 \\
\hline$M / I$ & $0.02(0.01)$ & $0.02(0.01)$ & 0.083 \\
\hline OGTT insulin AUC (pmol/L/120 min & $44,235.77(28,021.78)$ & $43,030.71(25,137.08)$ & 0.764 \\
\hline OGTT glucose AUC (mmol/L/120 min & $763.75(149.28)$ & $844.66(166.09)$ & $<0.001$ \\
\hline HOMA-IR & $12.58(2.27)$ & $10.69(0.19)$ & 0.092 \\
\hline
\end{tabular}

Data are, for categorical variables, given as frequencies (percentage) and for continuous variables as mean (standard deviation). $P$-values have been obtained as explained in the method section. FDR first-degree relatives, WHR waist-hip ratio, T2D type 2 diabetes, CHD coronary heart disease, HDL high-density lipoprotein, $L D L$ low-density lipoprotein, HOMA- $\beta$ homeostasis assessment model-beta, IGI insulinogenic index, DI disposition index, $M$ and $M / I$ insulin resistance level, GIR glucose infusion rate 
Table 2 Subject characteristics after matching by propensity score

\begin{tabular}{|c|c|c|c|}
\hline & Non-FDR & FDR & $\mathrm{p}$ \\
\hline $\mathrm{N}$ & 73 & 73 & \\
\hline Sex male (\%) & $33(45.2)$ & $35(47.9)$ & 0.868 \\
\hline Age (years) & $36.68(7.99)$ & $35.99(6.82)$ & 0.571 \\
\hline Weight (kg) & $83.93(14.86)$ & $81.83(14.71)$ & 0.391 \\
\hline $\mathrm{BMI}\left(\mathrm{kg} / \mathrm{m}^{2}\right)$ & $27.22(3.91)$ & $26.61(4.14)$ & 0.356 \\
\hline Fat percent (\%) & $27.87(9.24)$ & $27.18(8.69)$ & 0.647 \\
\hline $\mathrm{DI}(\mathrm{mL} / \mathrm{mol} / \mathrm{min})$ & $3.63(3.56)$ & $2.52(2.77)$ & 0.085 \\
\hline Waist circumference $(\mathrm{cm})$ & $90.37(10.64)$ & $92.15(11.59)$ & 0.343 \\
\hline WHR & $0.85(0.08)$ & $0.88(0.09)$ & 0.060 \\
\hline Diastolic blood pressure (mmHg) & $77.53(8.98)$ & $74.68(9.95)$ & 0.073 \\
\hline Systolic blood pressure $(\mathrm{mmHg})$ & $121.19(14.50)$ & $115.84(12.60)$ & 0.019 \\
\hline High heredity for T2D (\%) & $0(0.0)$ & $3(4.1)$ & $<0.001$ \\
\hline High heredity for CHD (\%) & $9(13.0)$ & $24(32.9)$ & 0.012 \\
\hline Currently smoking (\%) & $7(10.0)$ & $8(11.0)$ & 0.943 \\
\hline Exercise $>4$ times per week (\%) & $41(57.7)$ & $33(45.2)$ & 0.275 \\
\hline High stress level (\%) & $9(13.0)$ & $16(21.9)$ & 0.235 \\
\hline Serum adiponectin (ng/mL) & $8295.29(3551.68)$ & $7525.09(3707.54)$ & 0.242 \\
\hline Serum triglycerides (mmol/L) & $0.91(0.48)$ & $1.13(0.68)$ & 0.038 \\
\hline Serum HDL (mmol/L) & $1.42(0.32)$ & $1.47(0.36)$ & 0.418 \\
\hline Serum cholesterol (mmol/L) & $4.66(0.86)$ & $4.68(0.89)$ & 0.885 \\
\hline Serum LDL (mmol/L) & $3.78(0.80)$ & $2.59(0.67)$ & $<0.001$ \\
\hline Serum creatinine $(\mu \mathrm{mol} / \mathrm{L})$ & $74.91(12.45)$ & $88.44(15.75)$ & $<0.001$ \\
\hline OGTT fasting plasma glucose (mmol/L) & $4.74(0.36)$ & $4.85(0.49)$ & 0.123 \\
\hline OGTT plasma glucose $30 \mathrm{~min}(\mathrm{mmol} / \mathrm{L})$ & $7.55(1.54)$ & $8.15(1.39)$ & 0.017 \\
\hline OGTT plasma glucose $60 \mathrm{~min}(\mathrm{mmol} / \mathrm{L})$ & $6.90(1.90)$ & $7.99(2.24)$ & 0.002 \\
\hline OGTT plasma glucose $90 \mathrm{~min}(\mathrm{mmol} / \mathrm{L})$ & $5.87(1.51)$ & $6.97(1.99)$ & $<0.001$ \\
\hline OGTT plasma glucose $120 \mathrm{~min}(\mathrm{mmol} / \mathrm{L})$ & $5.56(1.37)$ & $6.11(1.61)$ & 0.032 \\
\hline OGTT fasting serum insulin (pmol/L) & $58.77(36.19)$ & $57.98(36.78)$ & 0.906 \\
\hline OGTT serum insulin $30 \mathrm{~min}(\mathrm{pmol} / \mathrm{L})$ & 489.05 (336.05) & $457.50(274.58)$ & 0.580 \\
\hline OGTT serum insulin $60 \mathrm{~min}(\mathrm{pmol} / \mathrm{L})$ & $454.58(297.68)$ & $498.54(314.31)$ & 0.429 \\
\hline OGTT serum insulin $90 \mathrm{~min}(\mathrm{pmol} / \mathrm{L})$ & $336.85(257.46)$ & $461.19(371.26)$ & 0.030 \\
\hline OGTT serum insulin $120 \mathrm{~min}(\mathrm{pmol} / \mathrm{L})$ & $305.38(267.73)$ & $401.78(380.42)$ & 0.103 \\
\hline HOMA- $\beta$ (\%) & $150.53(81.27)$ & $132.58(72.04)$ & 0.209 \\
\hline IGI $(\mathrm{pmol} / \mathrm{mmol})$ & $194.70(250.13)$ & $135.21(103.17)$ & 0.111 \\
\hline M-value (GIR/kg/min: g/kg/min) & $11.94(4.49)$ & $11.87(3.89)$ & 0.927 \\
\hline$M / I$ & $0.02(0.01)$ & $0.02(0.01)$ & 0.816 \\
\hline OGTT insulin AUC (pmol/L/120 min & $44,235.77(28,021.78)$ & $50,099.93(30,732.69)$ & 0.284 \\
\hline OGTT glucose AUC (mmol/L/120 min & $763.75(149.28)$ & $857.29(172.61)$ & 0.001 \\
\hline HOMA-IR & $12.58(8.36)$ & $12.81(8.67)$ & 0.879 \\
\hline
\end{tabular}

Data are, for categorical variables, given as frequencies (percentage) and for continuous variables as mean (standard deviation). $P$-values have been obtained as explained in the method section. FDR first-degree relatives, WHR waist-hip ratio, T2D type 2 diabetes, CHD coronary heart disease, $H D L$ high-density lipoprotein, $L D L$ low-density lipoprotein, HOMA- $\beta$ homeostasis assessment model-beta, IGI insulinogenic index, DI disposition index, $M$ and $M / I$ insulin resistance level, GIR glucose infusion rate 
A)



C)



B)

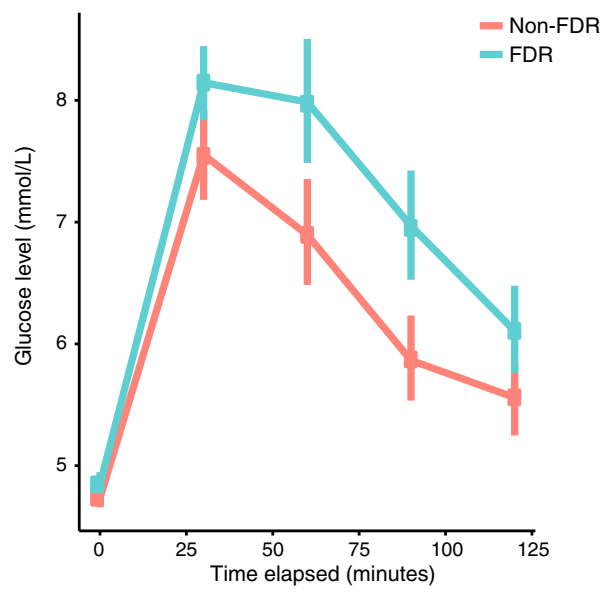

D)

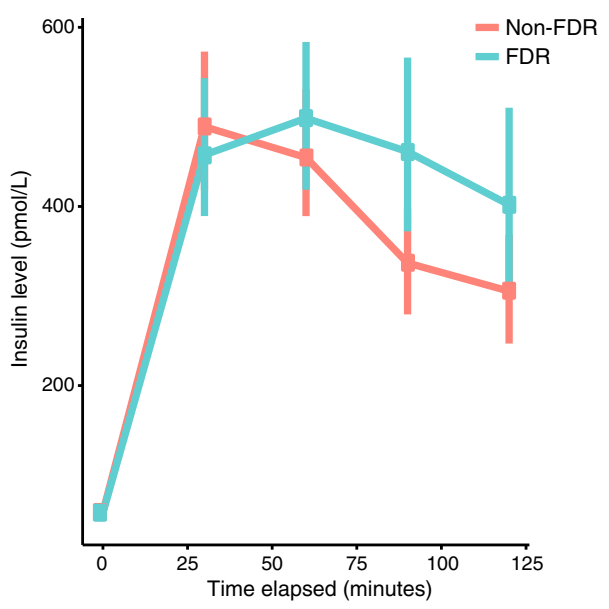

Fig. 1 a OGTT plasma glucose response in the overall set. OGTT = oral glucose tolerance test. AUC = area under curve. b OGT plasma glucose response in the matched set $(n=146)$. OGTT = oral glucose tolerance test. AUC = area under curve. c OGTT plasma insulin response in the overall set. OGTT = oral glucose tolerance test. AUC = area under curve. $\mathbf{d}$ OGTT plasma insulin response in the matched set $(n=146)$. OGTT = oral glucose tolerance test. $\mathrm{AUC}=$ area under curve

not statistically significant, in the FDR group, towards $\beta$ cell dysfunction, compared to the non-FDR. In our matched results, one could also see a tendency in insulin concentrations from the OGTT, that FDR had higher fasting insulin levels, lower $30 \mathrm{~min}$ values and then higher concentrations at 60, 90 and $120 \mathrm{~min}$. Possibly somewhat speculative, this further indicates $\beta$-cell dysfunction with a slow acute insulin response and a compensatory overproduction during the late response. In the previously mentioned study by Stadler et al., disturbances in insulin secretion (IGI) were also observed in the FDR group. They showed that acute phase insulin secretion was diminished and hyperinsulinemia during the late phase was present, despite normal insulin sensitivity [10]. Perseghin et al. showed that when subdividing the FDR into groups according to $\mathrm{M}$-value, the most insulin resistant individuals had a significantly lower acute insulin response than the normally insulin sensitive FDR, implying that the IR and $\beta$-cell dysfunction are indeed intimately connected, and more so than mere heredity [35]. Praveen et al. found that DI was lower in the FDR group than non-FDR, indicating $\beta$-cell dysfunction [13]. Gautier et al. concluded that FDR display early insulin secretion dysfunction during an IVGTT, despite normoglycemia [36]. Cnop et al. concluded that FDR's decline in glucose tolerance status is mostly due to a loss in insulin secretion ability [37].

Increasing BMI and fat percentage have often been associated with increasing insulin resistance. We confirmed these correlations in our study population, and found that in both groups, there was indeed a negative correlation between BMI and insulin sensitivity. In both 


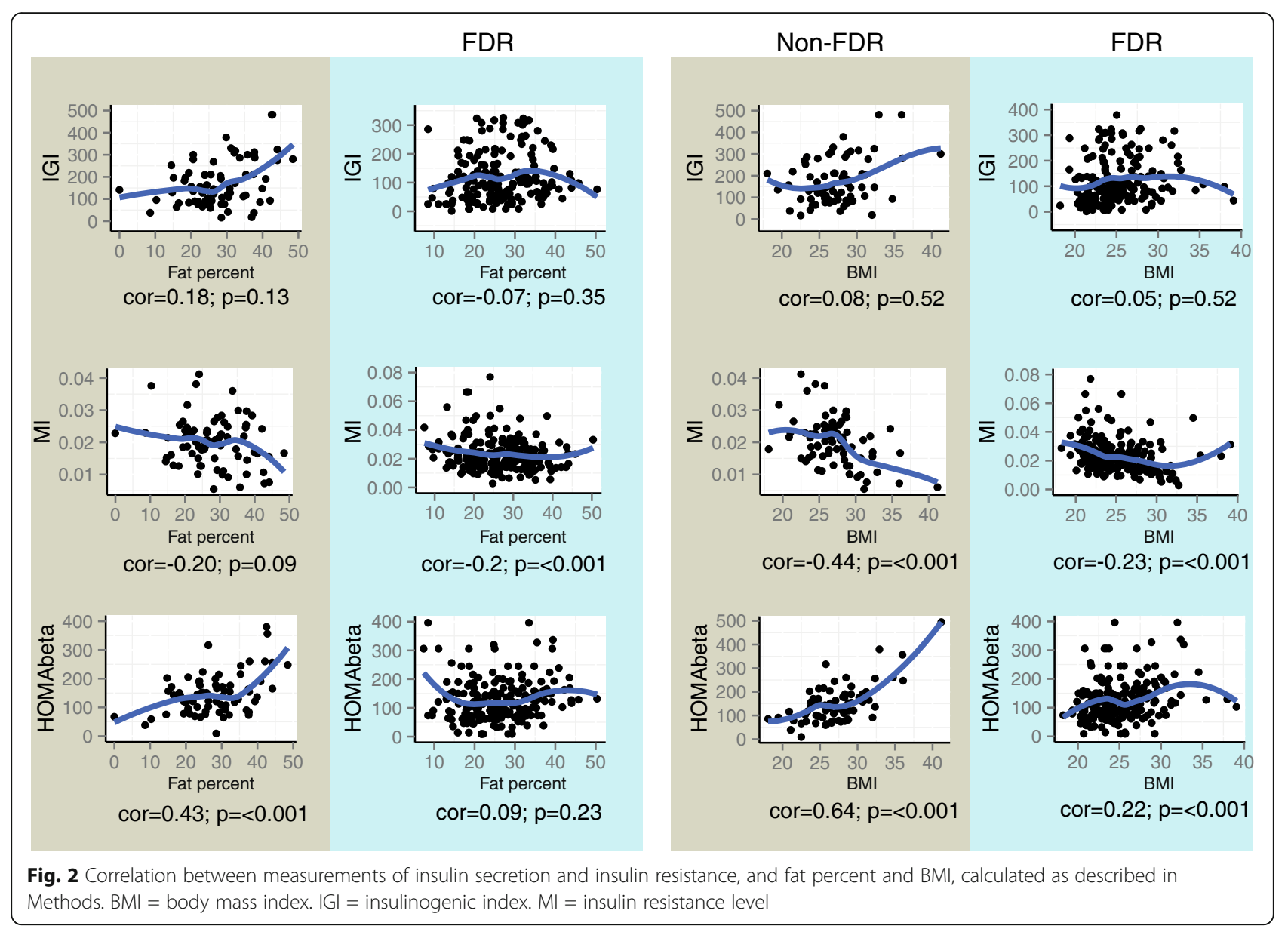

groups, increasing BMI was also associated with increasing HOMA- $\beta$, indicating a responding increase in insulin demand upon weight gain. However, the correlation coefficient was markedly lower among FDR than nonFDR, possibly indicating an inability to increase insulin production upon weight gain in the FDR group. Interestingly, we also found that among FDR, but not among non-FDR, increasing fat percentage was associated with decreasing MI, indicating an inability among the FDR to stay insulin sensitive upon expansion of the adipose tissue, specifically.

In our study, no differences in insulin resistance levels were seen, neither in adjusted nor unadjusted data. We hypothesize that this finding is possibly due to baseline differences between FDR and non-FDR, where the former had lower BMI. Other studies have shown both clamp data, $\mathrm{M}$ and $\mathrm{MI}$, and surrogate measures such as HOMA-IR to be deranges in the FDR group. Stadler et al., as well as Nyholm et al., showed that signs of IR (lower $\mathrm{M}$-value and $\mathrm{M} / \mathrm{I}$ ) were present in FDR when compared to a matched group of non-FDR [10, 32]. Praveen et al., as well as Kuzhandai et al. and Arner et al., displayed higher HOMA-IR, ie lower insulin sensitivity, in the FDR group than in non-FDR subjects $[13,14,38]$. Previous studies from our laboratory, performed on minor subgroups from the same study population but with more narrow inclusion criteria, have shown lower $M$ and/or $M / I$ values, as well as higher HOMA-IR and hypertriglyceridemia [38-40].

A major strength of this study was our usage of state of the art methodology to assess insulin sensitivity, as well as extensive measurements to assess $\beta$-cell function and adipose tissue function and biochemical data, including OGTT to assess glucose tolerance status and doing so in a relatively large study population [23, 27]. Other studies have looked at similar questions in similar study populations, however using less refined methodology [12-16].

Certain limitations to this study need to be addressed. In our results the non-FDR tended to be - not at a statistically significant level, however - more physically active, which could possibly have influenced our results. Also, we did not perform an IVGTT to assess $\beta$-cell function. In this study, we have not explored subjects' dietary habits. Information on diet could have contributed to increase the scientific value of our results, as a 
hyper-caloric diet is a well-established contributing factor in the development of obesity and T2D. Specific studies on the FDR group have also concluded that unhealthy dietary habits contributed to FDR's increased risk of cardiometabolic disease [2, 6, 41]. We did not include data on ethnicity, a known risk factor for glucose tolerance variations, in the two study groups. However, the vast majority of study participants were of Caucasian ethnicity, and we would thus not have had power to address if ethnicity was a contributing factor to differences between groups. Finally, among both groups were individuals with glucose tolerance disturbances, including one individual with overt T2D. However, the subjects were not included because they were normoglycemic, but because they were without known T2D and in general good health. We therefore have not performed any statistical analyses on subjects stratified by glucose tolerance status, as that in our opinion would make our data less representative of the background population. We consider it important to look at a population without too many modifications and excluding criteria, as that would make our sample less representative of the diverse background population.

Before matching, the FDR had lower mean BMI and less atherogenic blood lipid characteristics than the non-FDR. These differences in characteristics we believe were due to the non-FDR's higher weight, BMI and WHR, despite lower age. Mode of recruitment could have produced this bias, as we hypothesized that the FDR were aware of their higher risk of developing T2D, and thus more prone to be motivated to both participate in the study, despite normal weight, and possibly also to stay leaner and lead a healthier lifestyle. This could be considered a selection bias on our part, and a methodological imperfection. Smaller studies on subgroups from our study population, such as Axelsen et al. and Johanson et al., around 15 cases, plus controls, were recruited and then one can find subjects at matching BMI's $[39,42]$. In our populations, with almost 300 subjects, seeing leaner FDR and heavier non-FDR was to be expected. However, despite being significantly leaner, the FDR were less glucose tolerant, adjusted both by matching and least squares means, indicating a more unfavorable metabolic profile despite lower BMI than nonFDR. Furthermore, the non-FDR had a slightly lower WHR, not reaching statistical significance, possibly indicating a slightly more favorable regional adiposity. These results confirmed what Cederberg et al. hypothesized, that FDR are at an increased risk of T2D, obesity and specifically obesity-related insulin resistance [43]. As this study has included the recruited groups in their totality, taken from the background population and using minimal exclusion criteria, avoiding bias and confounders would be very difficult. We consider it important to include broadly from a background population to explore correlations and tendencies, as an important complement to longitudinal studies, and randomized clinical trials in particular, from which causative factors can be determined.

\section{Conclusion}

In this cross-sectional study of 200 FDR of T2D patients and 73 subjects without family history of T2D, we found that the FDR display glucose tolerance disturbances when compared to non-FDR, rather than insulin resistance. We also conclude that OGTT is a solid and informative, yet simple and inexpensive, mode of assessing risk of T2D in this high-risk group of FDR.

\begin{abstract}
Abbreviations
AIR: Acute insulin response; ALP: Alkaline phosphatase; ALT: Alanine aminotransferase; AUC: Area under curve; BMI: Body mass index $\left(\mathrm{kg} / \mathrm{m}^{2}\right)$; CHD: Coronary heart disease; Clamp: Euglycemic, hyperinsulinemic clamp; DI: Disposition index; FDR: First-degree relative; FFA: Free fatty acid; HDL: High-density lipoprotein; HOMA- $\beta$ : Homeostasis model assessment of the $\beta$-cell function index; IFG: Impaired fasting glucose;

IGI: Insulinogenic index; IGT: Impaired glucose tolerance; IR: Insulin resistance; IVGTT: Intravenous glucose tolerance test; LDL: Low-density lipoprotein; LIR: Late insulin response; M (M-value): Glucose infusion rate/ lean body mass during the euglycemic, hyperinsulinemic clamp; MI: Mvalue/insulin concentration during steady state of the euglycemic, hyperinsulinemic clamp; NGT: Normal glucose tolerance; OGT: Oral glucose tolerance test; T2D: Type 2 diabetes mellitus; WHO: World health organization; WHR: Waist/hip-ratio
\end{abstract}

\section{Acknowledgements}

The authors gratefully acknowledge the work of research nurses Carina Blomgren and Lena Silberberg for excellent patient care and Ida Sterner for technical assistance.

\section{Funding}

Co-author Björn Eliasson received funding from The Medical Training and Research Agreement, Region Västra Götaland to conduct the current study.

\section{Availability of data and materials}

The datasets used and analyzed during the current study are available from the corresponding author on reasonable request.

\section{Authors' contributions}

The authors have contributed equally to the manuscript. BE and $\mathrm{AH}$ participated in designing the study, recruiting subjects and collecting data. JH and AR performed the statistical analyses, and all authors interpreted and analyzed the results. $\mathrm{JH}$ drafted the manuscript, and all authors revised the manuscript.

\section{Ethics approval and consent to participate}

The protocol of study was approved by the regional Ethical Committee at the Sahlgrenska Academy at the University of Gothenburg (S 655-03) and was performed in agreement with the Declaration of Helsinki. All subjects received verbal and written information and gave written informed consent prior to inclusion in the study.

\section{Consent for publication}

Not Applicable.

\section{Competing interests}

The authors declare that they have no competing interests.

\section{Publisher's Note}

Springer Nature remains neutral with regard to jurisdictional claims in published maps and institutional affiliations. 
Received: 2 December 2016 Accepted: 30 June 2017 Published online: 14 July 2017

\section{References}

1. Defronzo RA. Banting lecture. From the triumvirate to the ominous octet: a new paradigm for the treatment of type 2 diabetes mellitus. Diabetes. 2009; 58(4):773-95.

2. Kahn SE, Cooper ME, Del Prato S. Pathophysiology and treatment of type 2 diabetes: perspectives on the past, present, and future. Lancet. 2014; 383(9922):1068-83.

3. Morris RD, Rimm DL, Hartz AJ, Kalkhoff RK, Rimm AA. Obesity and heredity in the etiology of non-insulin-dependent diabetes mellitus in 32,662 adult white women. Am J Epidemiol. 1989;130(1):112-21.

4. Zimmet P, Alberti KG, Shaw J. Global and societal implications of the diabetes epidemic. Nature. 2001;414(6865):782-7.

5. Fox CS, Coady S, Sorlie PD, D'Agostino RB Sr, Pencina MJ, Vasan RS, et al Increasing cardiovascular disease burden due to diabetes mellitus: the Framingham heart study. Circulation. 2007;115(12):1544-50.

6. Alberti KG, Zimmet P, Shaw J. International diabetes federation: a consensus on type 2 diabetes prevention. Diabet Med. 2007;24(5):451-63.

7. Defronzo RA, Tripathy D, Schwenke DC, Banerji M, Bray GA, Buchanan TA, et al. Prediction of diabetes based on baseline metabolic characteristics in individuals at high risk. Diabetes Care. 2013;36(11):3607-12.

8. Eckel RH, Grundy SM, Zimmet PZ. The metabolic syndrome. Lancet. 2005; 365(9468):1415-28.

9. Piatti P, Setola E, Galluccio E, Costa S, Fontana B, Stuccillo M, et al. Smoking is associated with impaired glucose regulation and a decrease in insulin sensitivity and the disposition index in first-degree relatives of type 2 diabetes subjects independently of the presence of metabolic syndrome. Acta Diabetol. 2014:51(5):793-9.

10. Stadler M, Pacini G, Petrie J, Luger A, Anderwald C. Beta cell (dys) function in non-diabetic offspring of diabetic patients. Diabetologia. 2009;52(11):2435-44.

11. Wagner R, Thorand B, Osterhoff MA, Muller G, Bohm A, Meisinger C, et al. Family history of diabetes is associated with higher risk for prediabetes: a multicentre analysis from the German Center for Diabetes Research. Diabetologia. 2013;56(10):2176-80.

12. Taheri N, Iraj B, Amini M, Amini P, Aminorroaya A. Cardiovascular risk factors in relatives of type 2 diabetics with normal glucose tolerance test and elevated one-hour plasma glucose. Endokrynologia Polska. 2010:61(4):359-63.

13. Praveen EP, Sahoo J, Khurana ML, Kulshreshtha B, Khadgawat R, Gupta N, et al. Insulin sensitivity and beta-cell function in normoglycemic offspring of individuals with type 2 diabetes mellitus: impact of line of inheritance. Indian J Endocrinol Metab. 2012;16(1):105-11.

14. Kuzhandai velu $V$, Jyothirmayi $B$, Kumar JS. Insulin resistance and alanine amino transaminase (ALT) levels in first degree relatives of type 2 diabetes mellitus. Diab Metabol Syndr 2011;5(3):143-147.

15. Ogedengbe SO, Ezeani IU. Profile of metabolic abnormalities seen in patients with type 2 diabetes mellitus and their first degree relatives with metabolic syndrome seen in Benin City, Edo state Nigeria. J Diab metabol Disord. 2014;13:61.

16. Amini $M$, Janghorbani $M$. Diabetes and impaired glucose regulation in firstdegree relatives of patients with type 2 diabetes in isfahan, iran: prevalence and risk factors. Rev Diabet Stud. 2007:4(3):169-76.

17. Han $X Y$, Ji LN, Zhou XH. Cross-sectional study of the pathophysiologic and clinical features in the first-degree relatives of type 2 diabetic patients. Beijing da xue xue bao Yi xue ban = J Peking Univ Health Sci. 2005;37(2):159-62

18. Friedewald WT, Levy RI, Fredrickson DS. Estimation of the concentration of low-density lipoprotein cholesterol in plasma, without use of the preparative ultracentrifuge. Clin Chem. 1972;18(6):499-502.

19. Definition and Diagnosis of Diabetes [cited 2014]. Available from http:// www.who.int/diabetes/publications/ Definition\%20and\%20diagnosis\%20of\%20diabetes_new.pdf.

20. Stolk RP, Orchard TJ, Grobbee DE. Why use the oral glucose tolerance test? Diabetes Care. 1995;18(7):1045-9.

21. Tai MM. A mathematical model for the determination of total area under glucose tolerance and other metabolic curves. Diabetes Care. 1994;17(2):152-4.
22. Matthews DR, Hosker JP, Rudenski AS, Naylor BA, Treacher DF, Turner RC. Homeostasis model assessment: insulin resistance and beta-cell function from fasting plasma glucose and insulin concentrations in man. Diabetologia. 1985:28(7):412-9.

23. Hanson RL, Pratley RE, Bogardus C, Narayan KM, Roumain JM, Imperatore G, et al. Evaluation of simple indices of insulin sensitivity and insulin secretion for use in epidemiologic studies. Am J Epidemiol. 2000;151(2):190-8.

24. Seltzer HS, Allen EW, Herron AL Jr, Brennan MT. Insulin secretion in response to glycemic stimulus: relation of delayed initial release to carbohydrate intolerance in mild diabetes mellitus. J Clin Invest. 1967;46(3):323-35.

25. Gastaldelli A, Ferrannini E, Miyazaki Y, Matsuda M, DeFronzo RA. Beta-cell dysfunction and glucose intolerance: results from the San Antonio metabolism (SAM) study. Diabetologia. 2004;47(1):31-9.

26. Ferrannini E, Mari A. Beta cell function and its relation to insulin action in humans: a critical appraisal. Diabetologia. 2004:47(5):943-56.

27. Ferrannini E, Mari A. How to measure insulin sensitivity. J Hypertens. 1998; 16(7):895-906.

28. DeFronzo RA, Tobin JD, Andres R. Glucose clamp technique: a method for quantifying insulin secretion and resistance. Am J Phys. 1979;237(3):E214-23.

29. Altman DG. Practical statistics for medical students. London: Chapman \& Hall; 2000.

30. Kirkwood BE, JACS. Medical Statitics. Oxford: Blackwell Publishing: 2003.

31. Wahlgren L. SPSS steg för steg. Lund: Studentlitteratur; 2005.

32. Nyholm B, Nielsen MF, Kristensen K, Nielsen S, Ostergard T, Pedersen SB, et al. Evidence of increased visceral obesity and reduced physical fitness in healthy insulin-resistant first-degree relatives of type 2 diabetic patients. Eur J Endocrinol. 2004;150(2):207-14

33. Alyass A, Almgren P, Akerlund M, Dushoff J, Isomaa B, Nilsson P, et al. Modelling of OGTT curve identifies $1 \mathrm{~h}$ plasma glucose level as a strong predictor of incident type 2 diabetes: results from two prospective cohorts. Diabetologia. 2015;58(1):87-97.

34. Metter EJ, Windham BG, Maggio M, Simonsick EM, Ling SM, Egan JM, et al. Glucose and insulin measurements from the oral glucose tolerance test and mortality prediction. Diabetes Care. 2008;31(5):1026-30.

35. Perseghin G, Ghosh S, Gerow K, Shulman Gl. Metabolic defects in lean nondiabetic offspring of NIDDM parents: a cross-sectional study. Diabetes. 1997:46(6):1001-9.

36. Gautier JF, Wilson C, Weyer C, Mott D, Knowler WC, Cavaghan M, et al. Low acute insulin secretory responses in adult offspring of people with early onset type 2 diabetes. Diabetes. 2001;50(8):1828-33.

37. Cnop M, Vidal J, Hull RL, Utzschneider KM, Carr DB, Schraw T, et al. Progressive loss of beta-cell function leads to worsening glucose tolerance in first-degree relatives of subjects with type 2 diabetes. Diabetes Care. 2007:30(3):677-82

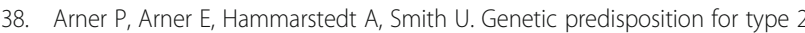
diabetes, but not for overweight/obesity, is associated with a restricted adipogenesis. PLoS One. 2011;6(4):e18284.

39. Axelsen M, Smith U, Eriksson JW, Taskinen MR, Jansson PA. Postprandial hypertriglyceridemia and insulin resistance in normoglycemic first-degree relatives of patients with type 2 diabetes. Ann Intern Med. 1999:131(1):27-31.

40. Henninger AM, Eliasson B, Jenndahl LE, Hammarstedt A. Adipocyte hypertrophy, inflammation and fibrosis characterize subcutaneous adipose tissue of healthy, non-obese subjects predisposed to type 2 diabetes. PLoS One. 2014;9(8):e105262.

41. Adamson AJ, Foster E, Butler TJ, Bennet S, Walker M. Non-diabetic relatives of type 2 diabetic families: dietary intake contributes to the increased risk of diabetes. Diabet Med. 2001;18(12):984-90.

42. Johanson EH, Jansson PA, Gustafson B, Lonn L, Smith U, Taskinen MR, et al. Early alterations in the postprandial VLDL1 apoB-100 and apoB-48 metabolism in men with strong heredity for type 2 diabetes. J Intern Med. 2004:255(2):273-9.

43. Cederberg H, Stancakova A, Kuusisto J, Laakso M, Smith U. Family history of type 2 diabetes increases the risk of both obesity and its complications: is type 2 diabetes a disease of inappropriate lipid storage? J Intern Med. 2015: 277(5):540-51 